\title{
Género y trabajo social: algunos desafíos
}

\author{
Gender and Social Work. Some Challenges
}

MÓNICA DE MARTINO

Universidad de la República del Uruguay

RECEPCIÓN: 8/I I/2OI2 · ACEPTACIÓN: I3/O5/20I3

RESUMEN El presente artículo es un subproducto de la investigación titulada "Ser hombres, ser padres en contextos de pobreza", desarrollada en el marco del posdoctorado en el Programa de Doctorado en Ciencias Sociales Interdisciplinarias de la Facultad de Ciencias Sociales de la Universidad Federal de Santa Catarina. Si bien la investigación apunta al campo de los estudios sobre masculinidades, es necesario detenerse en la amplia bibliografía sobre género producida en las últimas décadas para comprender los dilatados debates en torno a tal categoría. Sin intención de agotarla y sin centrarse específicamente en los estudios sobre masculinidades, el presente artículo intenta establecer una interpretación posible —entre otras- sobre la evolución teórica del concepto. Pensamos que la evolución en las formas de interpretación del mismo y las formas de debatirlo podrían apuntar a una posible fetichización o cosificación del propio concepto. Para fundamentar tal proposición colocaremos en debate a las filósofas Simone de Beauvoir y Judith Butler, como exponentes de dos corrientes epistemológicas que marcan posturas sobre la temática. A saber: una postura materialista-existencial y otra que puede enmarcarse en lo que ha sido denominado como constructivismo radical. A parte de ellas, desde el campo de los estudios sobre masculinidades retomaremos la propuesta de Connell, quien aporta una mirada diferente, trayendo a la luz las categorías de interés y capacidad heurística. A partir de estos autores intentaremos señalar algunos desafíos para 
el trabajo social, aunque los mismos pueden involucrar al conjunto de las profesiones asistenciales. Tal vez los más notorios sean: i) la ausencia de una reflexión epistemológica a la hora de afiliarse a ciertas posturas hoy en boga; y ii) la ausencia también de una perspectiva de género a la hora de intervenir en diferentes campos de actuación. Esta última es aún más paradójica en la medida que contamos con un cuerpo profesional altamente feminizado.

PALABRAS CLAVE Género, materialismo, constructivismo radical, trabajo social.

ABSTRACT This article is a product of the research entitled Be Men, Be Fathers in the context of poverty, developed under the postdoc in the PhD Program of the Federal University of Santa Catarina. While the research points to the field of men's studies is necessary to stop the extensive literature on sex and gender in recent decades product to understand the lengthy discussions over this category. Without intending to exhaust, without focusing specifically on masculinity studies, this article attempts to establish a possible interpretation, among others - on the theoretical evolution of the concept. We think that the evolution in the interpretation of the ways and forms of debate may point to a possible fetishization or reification of the concept. In support of this proposition will place in the debate to philosophers Simone de Beavoire and Judith Butler, as exponents of two currents which mark epistemological positions on the issue. Namely, a materialist - existential position and one that can be framed in what has been called radical constructivism. From the field of men's studies took up the proposal for Connell, who brings a different perspective, bringing to light categories of interest and heuristic. From these authors try to point out some challenges for social work, although they may involve the whole of the caring professions. Perhaps the most notable are: (i) the absence of an epistemological reflection when joining certain viewscurrently in vogue, and (ii) the absence also of a gender perspective when intervening in different fields of action. The latteris even more paradoxical to the extent that we have a highly feminised professional body.

KEYWORDS Gender, materialism, radical constructivism, social work. 


\section{Introducción}

Como se adelanta en el resumen, intentamos aportar algunos elementos sobre cómo el concepto género se encuentra involucrado con el trabajo social en términos de determinados desafíos teórico-metodológicos. Para ello, sin la intención de agotar la bibliografía existente sobre sexo y género, en un primer momento colocaremos en debate algunos de las/los autoras/es que consideramos han realizados esfuerzos superlativos para comprender el género, claro está, desde posturas epistemológicas muy diversas. En la medida que realizamos este diálogo triangulado, deslizaremos ciertas líneas de interpretación sobre la propia categoría género y sobre su incidencia en el trabajo social.

Vale la pena recordar que en décadas pasadas, especialmente a partir de los años setenta, el género asumió el carácter de concepto analítico central tanto para el estudio sobre la condición de la mujer como para el accionar de grupos y proyectos interdisciplinarios y también políticos. Si bien siempre tuvo la condición de ser una categoría estrictamente gramatical que habilitaba subdivisiones dentro de una clase, comenzaron a imputársele otros contenidos asociados a características culturalmente específicas de la masculinidad y la feminidad, es decir, comenzó a implicar caracteres culturales imputados a cuerpos sexuados.

En primer lugar, el uso del concepto género permitió rechazar el determinismo biológico haciendo visible la amplia gama de construcciones culturales de lo femenino y lo masculino. Las discusiones sobre género en distintas esferas se ha convertido en una característica del saber contemporáneo. Las décadas de los setenta y los ochenta han sido generosas en términos de pensar el género. Pero creemos que no todos los debates sobre género han impactado en nuestro colectivo profesional. En líneas generales, algunos de ellos han llegado a estos lares a través de difusoras básicamente latinoamericanas. Son escasas/os las/os autores cuyas obras han sido recepcionadas de manera directa por los/las trabajadores/as sociales. Por lo tanto, pensamos que nuestra profesión se alimenta, siempre a modo de tendencia, de teorías de segunda generación, por decirlo de alguna manera. Esto deviene en una apropiación acrítica y disolvente de las diferentes formas de abordar este concepto.

$\mathrm{Al}$ adoptar una perspectiva de género, si este es el caso, el trabajo social deberá también interrogarse respecto a dos cuestiones que consideramos fundamentales: i) ¿puede el género ser considerado una herramienta analítica que disipa cualquier postura natural y naturalizante al respecto?; y ii) ¿puede ser 
abordado como explicación totalizadora? Para explorar estas preguntas, este artículo investiga y coloca en debate esfuerzos por teorizar el género.

\section{Judith Butler versus Simone de Beauvoir}

\section{En nombre de la madre}

Ya es sumamente conocida la posición de Beauvoir respecto al género, plasmada en la reiterada frase: «nadie nace mujer, se hace mujer». Pero se hace mujer en una situación particular que por definición es el lugar fáctico donde la libertad, en tanto plenitud, puede ejercerse sólo de manera limitada. Todo sujeto ni es absoluto ni tiene libertad absoluta: se trata de un sujeto en interacción con otros, intrínsecamente libre pero también socialmente construido y limitado. Para el caso de la mujer, los límites impuestos por su situación en tanto mujer son paradigmáticamente ilustrativos. Subordinada y expuesta a la opresión, la mujer es objetivada, en tal situación, como lo otro (Femenías, 2000).

Lo otro no es una categoría ontológica sino cultural, por tanto modificable. $\mathrm{Al}$ respecto, el análisis que realiza sobre el cuerpo de las mujeres y de la maternidad nos habla de ese otro culturalmente modificable. Beauvoir desmitifica la maternidad al señalar que las mujeres se reconocen socialmente más como madres que como mujeres por el vínculo singular que entablan madres e hijos y por el olvido de sí que la maternidad implica (Femenías, 2000). Abrirá nuevas puertas al legitimar el no deseo de ser madre o la opción por la interrupción del embarazo, indicando la necesidad de que las mujeres se apropien de su cuerpo y de las decisiones en torno a él. Al respecto, si bien acepta una base biológica del dimorfismo sexual, reconoce que puede elegirse legítimamente otro del propio sexo como objeto de deseo. No adopta, por tanto, respecto a la biología una postura determinista. Si para la autora el sexo es ante todo sexo vivido, es decir, vivido culturalmente, nadie puede entonces situarse más allá de su sexo. En este sentido, el cuerpo es el locus de las experiencias vividas concretamente, es el cuerpo-sujeto en situación (Femenías, 2000).

Podríamos resumir burdamente su postura indicando que es la sumatoria de un universal (su concepto de sujeto trascendente), particularizado en un cuerpo sexuado y oprimido (mujer). Teórica y metodológicamente, el género, el devenir mujer u hombre con cierta orientación sexual, es una mediación más del proyecto que define a ese sujeto, tal como posteriormente lo definirá Sartre en el apéndice a su Crítica a la razón dialéctica, denominado "Cuestiones de método». 
Los feminismos estructuralistas y/o constructivistas radicalizados de los sesenta y setenta parten de esta concepción de cuerpo situado y - mal que les pese- Beauvoir se convirtió en una suerte de condición de posibilidad de su existencia. Al decir de Staton ( I980), las feministas posestructuralistas radicales norteamericanas que recepcionaron la obra de la autora francesa, lo hicieron a partir de una desconexión transatlántica, es decir, sin una apropiación del corpus de la filosofía existencialista ni de la obra de la autora in totum. Para confrontar ideas, seleccionamos las elaboraciones de Judith Butler, representante de ese constructivismo radical, poskantiano y poshegeliano, del cual se han apropiado de manera acrítica nuestra profesión y otras también vinculadas al trabajo socio-asistencial (Femenías, 2000).

Para Butler, las elaboraciones de Beauvoir indicarían que ser mujer es un acto de voluntad cultural, una construcción que designa los variados modos del proceso de autoconstrucción del género mujer, a lo que se suma que sólo existiría ese género. Si el cuerpo era un cuerpo situado, el ser mujer es, entonces, en ese proceso de autoconstrucción, una interpretación cultural del cuerpo hembra. Llegar a ser mujer es un proceso propositivo de autoconstrucción y de apropiación de un conjunto de actos y de habilidades que encuadra arbitrariamente en el concepto de proyecto sartreano, a la manera de la asunción de un cierto estilo y de ciertos significados corporales, como un trabajo consciente de incardinamiento (embodyment). Por tanto, a su juicio, Beauvoir ignora en sus análisis el objetivo performativo del género sobre el sexo, que se construye excluyendo ciertas posibilidades e invisibilizando otras. Entonces, este llegar a ser el propio género debe ser entendido como un proceso de elección que nos lleva a donde siempre estuvimos, en una circularidad asfixiante: nacimos hembras-nos hacemos mujeres, siempre en el eterno femenino (Femenías, 2000).

El libro Gender Trouble de Butler nos informa acerca de cómo la «naturalidad» de sexo, sexualidad y género está «constituida a través de actos performativos discursivamente constreñidos que producen el cuerpo a través y dentro de las categorías de sexo" (I990: x). Butler advierte al principio que «ser un sexo o un género es fundamentalmente imposible» (I990: I9). Las oposiciones binarias hombre/mujer y masculino/femenino son incompatibles con la variabilidad y complejidad de las características humanas, y construyen no sólo una falsa oposición entre los sexos, sino también una homogeneización artificial dentro de cada término de ese par binario. Los géneros estereotípicos deben entenderse como "señales ontológicas que son fundamentalmente inhabitables» (I990: I46). Rechazando el «viejo sueño de la simetría», Butler 
argumenta que el género se debe entender no como un conjunto de atributos, sino como un hacer, una reiteración de conductas, una performance, que constituye la identidad que da a entender qué se es (I990: 24).

Para la autora, el género es el proceso que construye esa supuesta coherencia interna del sexo, deseo (hetero)sexual y práctica (hetero)sexual dentro del sujeto moderno. Es el mecanismo que permite pensar un «cuerpo presocial» moldeado por la cultura y que proporciona un criterio de inteligibilidad para las personas que inspira tanto el paradigma naturalista como el paradigma del yo, tan caro este último a Beauvoir.

Género es el medio discursivo/cultural mediante el cual se produce la naturaleza sexuada o un «sexo natural» y se establece como un «prediscursivo", anterior a la naturaleza, una superficie políticamente neutral sobre la cual actúa la cultura (Butler, I990: 7).

El género realiza este trabajo de naturalización de la «repetición estilizada de acciones a través del tiempo (Butler, I990: I4I). O sea, la actitud «natural» se produce a través de la repetición de palabras, actos y gestos. Tales reiteradas repeticiones llevan a concebir la «naturalidad» del cuerpo y de la heterosexualidad y a actuar de acuerdo con los «dictados de la naturaleza». El género funciona, pues, como un padrón regulatorio, «una fabricación, una fantasía instituida e inscrita en la superficie de los cuerpos» (Butler, I990: I36).

Pero Butler fuerza a Beauvoir. Para la autora francesa, la libertad incardinada, el sujeto como proyecto, sugiere la indistinción del sexo/género, subsumidos en la noción de cuerpo vivido. Es decir, experimentado como el cuerpo que yo soy.

Butler interpreta a su manera la noción de hacerse, en el sentido que en el marco filosófico sartreano el género sería también parte del proyecto, una elección impregnada de los resabios cartesianos del sujeto intencional, tributario del modelo humanista, de un pensamiento materialista, que Butler rechaza (Femenías, 2000: 36).

Pero para Beauvoir el género es a su vez construcción cultural y elección. Las mujeres son existencia, el eterno femenino es una mera construcción de la cultura dominante, producto de un mundo masculino que las heterodesigna. El otro no es ni recíproco ni simétrico con los varones. Esa es su peculiaridad, que siendo libertad, en tanto humanas, quedan definidas como inesenciales, 
inmanentes y polo de alteridad, solamente. Al respecto, para Butler las mujeres de Simone han antepuesto su condición de mujeres a su condición de humanas, lo que sería inconcebible para Beauvoir, para quien cada mujer debe identificarse a sí misma como un ser humano, que además es mujer y se inserta en una situación totalmente diferente a la de los varones (Femenías, 2000).

\section{Aportes desde los estudios sobre masculinidades}

El libro Gender and Power de R. W. Connell (I987) se identifica con tendencias neomarxistas, existencialistas y posestructuralistas, lo que lo hace un autor referente. ${ }^{1}$ Connell expone una «teoría social sistemática del género» que lucha por representar su historicidad; el papel dinámico del género en el plano del trabajo, el poder y la catexis. Intenta, además, vincular agencia y estructura social en la formación y reproducción del género, así como la turbulencia y las contradicciones que pertenecen al género como experiencia vivida. Como él mismo expresa, desarrolla una teoría del género «basada en la práctica», atenta tanto al poder limitante del género como a los intentos de liberarse de tales limitaciones.

Existen, obviamente, líneas que lo separan de Butler: el género como una práctica social es algo más que una simple marca del cuerpo humano, «es el tejido de una estructura de símbolos que exageran y distorsionan el potencial humano» (1987: 79). Se opone al dualismo mente/cuerpo, insistiendo en que «la transformación práctica del cuerpo en la estructura social del género no se realiza sólo a nivel del simbolismo: tiene efectos físicos en el cuerpo, la incorporación es material» (I987: 87).

Para construir una mirada histórica sobre el género o, en otras palabras, analizar la historicidad del mismo, Connell apela a Marx y Sartre para delinear una concepción de las prácticas humanas en relación con las estructuras sociales. Según esta perspectiva, las prácticas son las acciones diarias de los seres humanos que se apropian de y transforman la naturaleza para satisfacer sus necesidades y, en el proceso, transformarse a sí mismos, produciendo nuevas necesidades y nuevas prácticas. Las prácticas también pueden ser definidas como transformaciones sobre el mundo natural que abren nuevas posibilida-

I. Si bien Connell es actualmente una mujer transgénero, utilizamos el masculino en la medida que las obras citadas corresponden a su trayectoria académica como hombre. 
des, así como nuevos riesgos y presiones. También pueden instituirse e institucionalizarse, creando un grado de disciplina en el mundo social que limita la libertad de futuras prácticas. Connell define la estructura social en términos de esos límites:

Una estructura social es un padrón de constreñimiento sobre la práctica inherente a las relaciones sociales. Aunque las estructuras marcan la fijeza del mundo social, la sedimentación de prácticas pasadas que limitan la acción presente, la dimensión de la vida colectiva que existe más allá de la intención individual, no son impermeables al cambio.

La práctica se puede volver contra lo que la constriñe; así la estructura puede ser deliberadamente el objeto de la práctica. Pero la práctica no puede eludir la estructura, no puede flotar libre de sus circunstancias (Connell, I987: 95).

Como mejor puede entenderse el género es como un conjunto interrelacionado de estructuras sociales que organizan la vida social en torno al sexo y la sexualidad. Es más, el autor argumenta que el género puede concebirse en términos de estructuras muy específicas vinculadas a prácticas sociales particulares de trabajo, poder y catexis, es una estructura autónoma constitutiva de esos campos. Así, por ejemplo, en el ámbito del trabajo, opera como una limitante al definir la división sexual del trabajo, la organización de la vida doméstica, la división entre trabajo pagado y no pagado, etcétera. Dentro del ámbito del poder, el género estructura la autoridad, el control y la coerción, bajo el monopolio masculino del ejercicio de la violencia. En el plano de la catexis, nos habla de prácticas que construyen relaciones emocionalmente, en las que el género estructura sujetos deseantes y objetos deseables, padrones de deseo y prácticas sexuales, así como los términos y condiciones del intercambio sexual. Estas estructuras de género existen en una interrelación compleja que denomina composición histórica. El género así entendido es «un concepto vinculante. Se trata de vincular otros campos de la práctica social con prácticas modales de generización» (Connell, I987: I40).

Forzando la comparación, tal vez para la creación y transformación del género, sea un punto común entre las posturas aquí analizadas la importancia de la agencia, en términos de Connell, la performance según Butler o el proceso de devenir mujer de acuerdo a Beauvoir, algo que aquella postura natural no incorpora. No obstante, si Beauvoir y Connell expresan diferentes posturas 
materialistas, Butler se extravía en un constructivismo radical que hace sinónimos al sexo y al género. Su apuesta es a la intelección de los problemas de las minorías sexuales. Intelección radical, en tanto pérdida de la materialidad corpórea y del reconocimiento de las diferencias.

Y si Beauvoir hace gala de la comprensión de un sujeto trascendental singularizado en un cuerpo de hembra que se transforma en un particular -esa hembra que devino mujer- Connell coloca énfasis en los constreñimientos de la estructura sobre la agencia y en la necesidad de explorar nuevas formas de pensar el género de manera tal que diferentes esferas de lo social puedan ser analizadas a partir de esta perspectiva teórica no totalizadora.

\section{Prácticas profesionales y producción social de vocaciones}

No debemos olvidar que muchas de las profesiones asistenciales, entre ellas el trabajo social, son, casi por definición, altamente feminizadas. Si analizamos históricamente a la profesión, la continuidad de la presencia femenina de las protoformas del servicio social hasta su formato profesional, hace que se desdibujen las propias determinaciones de la institucionalización profesional. Desde las damas de la caridad a los agentes profesionales parece haber habido una «evolución» por acumulación de conocimientos y técnicas en la población femenina de ciertos segmentos sociales (Piriz, I994).

Cabe entonces preguntar junto a Grassi (2005: Io) cuando interroga al trabajo social argentino:

¿Significa esto que es el género de sus agentes [...] lo que determina las características de este campo profesional y su lugar relativamente subordinado entre las disciplinas de lo social? ¿O es más conducente distinguir las preguntas referidas a la profesión y la construcción de su objeto de intervención, de aquellas que se refieren a la producción social de las vocaciones y la «natural» elección de carreras, que atrae a las mujeres al trabajo social y aleja a los varones?

Tal vez ambos componentes de estas interrogantes nos permitan comprender la feminización de ciertas profesiones y/o de algunas de sus especializaciones. Debemos remontarnos al siglo XIX, cuando el higienismo pautó la constitución del surgimiento del campo de muchas de las profesiones, digamos, modernas. Paralelamente la mujer quedaba relegada a su papel de enfermera, monja o visitadora social. Si estas coacciones caracterizaron la primera etapa 
de algunas profesiones, luego las mujeres las «eligieron», pero a partir de vocaciones producidas socialmente con base en atributos imputados arbitrariamente a las mujeres: ayuda, colaboración, cuidados, profesiones que permiten conciliar una doble o triple jornada, etcétera (Barros Cassal y Abrahão da Silva, 20ıо; Guiliani, 2006; Rebollo, González y García, s/f).

Existen dos fuentes de debilidad para el trabajo social respecto a su sensibilidad ante una perspectiva de género. En primer lugar, una inherente al propio concepto: nos permite conocer en parte al cuerpo profesional y, en menor medida, nos permite conocer sus prácticas y sus objetos de intervención. Prácticas y objetos en general asistenciales, difusos y compulsivos, que colocan en juego a «la madre interior» que toda mujer que se precie lleva dentro (Chodorow, I978; Piriz, I994). En segundo lugar, otra inherente al habitus ${ }^{2}$ profesional: el remitirse a textos de «segunda generación», ignorando la génesis de teorías, las posturas de autores con relación a determinadas escuelas, etcétera.

Para superar estas fuentes de debilidad, el trabajo social deberá construir un conjunto de categorías que permitan definir y explicar sus procesos y procedimientos y sus mutuas y específicas relaciones. No así con las categorías estrictamente teóricas con las que trabaja, pues ellas provienen del amplio campo de las denominadas ciencias sociales. A partir de ello, podrán colocarse en un lugar de autoridad e interdisciplinariedad y no en un lugar subordinado que implica la necesidad de defender espacios, hacerse visible, así como las mujeres en sus luchas políticas (Grassi, 2005).

Lo interesante, en líneas hipotéticas, es que tal vez ese lugar de subordinación del trabajo social se encuentre relacionado con su propia feminización. Pero lo que queda claro es que aquel desafío no puede entenderse solamente bajo estas condiciones. La defensa de un cuerpo profesional feminizado, asociado a las luchas feministas, no es la única condición de posibilidad del surgimiento de prácticas profesionales entendidas «como unidad reflexiva de acciones con fundamento, referida a problemas de cuya definición [...] deberá(n) ser partícipe(s) y no 'consumidor(es)' pasivos» (Grassi, 2005: I2).

Deriva de ello que, en términos de género, un segundo desafío lo constituye superar la ausencia clara de cualquier perspectiva de género. O la incorporación ingenua, empirista y no relacional del concepto: se trabaja la cuestión femenina o la cuestión masculina, se trabaja con mujeres o con hombres. El tra-

2. Entendemos la categoría habitus como la ha trabajado Pierre Bourdieu a lo largo de su obra. 
bajo social, y tal vez otros colectivos profesionales, se encuentran impregnados, por defecto, por lo que Garfinkel (1967) ha denominado una actitud natural hacia el género, es decir, por una postura basada en un empirismo naturalista que necesita ser superado. Tal actitud natural, más allá de los discursos y a la hora de analizar las prácticas profesionales, incorpora una serie de axiomas dicotómicos no cuestionables sobre la categoría género: existen dos géneros, sólo dos que son invariables, la genitalidad es el signo esencial del género, etcétera. Superar este empirismo naturalista es un emprendimiento fundamental. Desnaturalizar lo naturalizado y asumido profesionalmente (Piriz, I994).

En segundo lugar, derivaría de lo anterior que, para muchos profesionales, la naturaleza del género sigue siendo mera evidencia, reforzando esa postura natural hacia el mismo. Y lo hacen abrevando teóricamente en las ricas concepciones producidas, especialmente, a partir de la mitad del siglo XX, que indican que el concepto se ha tornado en algo sumamente controvertido tanto en el ámbito de las teorías feministas como en el de los estudios sobre masculinidades. El problema radica en los niveles de apropiación de los debates teóricos relativos a género. A este tipo de profesión no le correspondería producir teoría al respecto, sino buscar las formas más apropiadas para instrumentalizarlo a nivel de prácticas profesionales.

Si observamos esta proliferación de autores/as y las diferentes formas de entender el género, podríamos indicar, a modo de hipótesis, que existe una tendencia disolvente del concepto. $\mathrm{O}$, en otras palabras, identificamos una tendencia hacia la cosificación o fetichización del mismo. Parecería ser que puede explicar todo y que puede ser explicado también desde todas las perspectivas y esto no es problematizado a la hora de la intervención o a la hora de pensar el objeto de conocimiento e intervención.

En aras de no hipostasiar una de las tantas dimensiones que explican lo que sucede entre hombres y mujeres y entre agencia sexuada y estructura social, es que hablamos de una fetichización del concepto que, además, ha sido asociado básicamente a la condición femenina, condición de las mujeres o estudios sobre la mujer. Esta feminización e incardinación del concepto es lo que también lo transforma, teóricamente pero también desde una perspectiva de la práctica, en un fetiche: género es igual a mujer y su condición, aún en plural, cuando teóricamente ningún concepto es, por definición, sexuado.

Creemos que este es un problema fundamental en y para las prácticas profesionales. Con una postura natural, y alimentados de teorías de segunda generación, es muy difícil apropiarse de la génesis del concepto y de las escuelas 
teóricas que se conjugan en él. Este fetiche es el que, en líneas generales, es utilizado a la hora de la intervención.

Para definir esta tendencia a la cosificación del concepto nos hemos basado en otras elaboraciones. Ya algunas feministas han planteado algunas dudas sobre la utilidad del género como categoría analítica. Bordo ha identificado dos corrientes que alimentan lo que ha llamado un nuevo «escepticismo sobre el género» (I993: 216). Una corriente proviene de las experiencias de feministas de raza negra y de feministas lesbianas que han sugerido que el «riesgo múltiple», característico de sus vidas, plantea serias preguntas sobre la validez de las generalizaciones del género. Si siempre está mediado por la raza, la clase, la etnia, la orientación sexual, entonces un marco referencial que aísla el género, que lo construye en términos de "modelo aditivo" tiene graves fallas (King, I988; Spelman, I988). La otra corriente emana de ciertas críticas posmodernas que describen las narrativas de género totalizadoras y que crean una falsa homogeneidad a partir de colectivos - mujeres u hombres- que son básicamente heterogéneos. Además han puesto en duda la oposición binaria que fija a hombres y mujeres en sus cuerpos, desafiando así la base de la distinción sexo/género.

Si el género si inventó para iluminar la construcción social de la masculinidad y la feminidad y de manera ingenua dio por supuesto el cuerpo sexuado, entonces tiene poco que ofrecer en un mundo posmoderno que entiende el cuerpo, el sexo y la sexualidad como socialmente construidos (Hawkesworth, I999: 6).

Para aportar a esa construcción de procesos, procedimientos y relaciones, necesaria para la construcción de campos profesionales autónomos, atravesados por el género y por la lucha de las mujeres — cosas diferentes, por cierto-, mencionamos a continuación algunos puntos que consideramos desafiantes para las prácticas profesionales rutinarias.

\section{Género y prácticas profesionales rutinarias}

\section{La producción de los sexos}

Si hay algo en común, más allá de las diferencias epistemológicas, entre los autores brevemente reseñados es la importancia otorgada a las relaciones de poder, a las instituciones, prácticas y discursos que establecen y regulan la for- 
ma y significado del género. En otras palabras, los dispositivos que permiten que los sexos sean establecidos. Cabe preguntarse, entonces, cuáles prácticas producen género. Podríamos enumerar algunas: el falocentrismo, la norma que establece como padrón normativo la heterosexualidad, el tabú del incesto. Todas estas concepciones internalizadas naturalizan la agencia sexual masculina, la colocan como padrón que: i) marca la diferencia (Butler, I990); ii) la diferencia y la opresión (Beauvoir); iii) el constreñimiento de las prácticas del agente en un orden social dado (Connell, I987).

Tales prácticas funcionan en un complejo circuito de prohibición, producción y naturalización del sexo/género/deseo y es en ese circuito donde se insertan las prácticas profesionales, ya sea para reproducirlo o para -raramente- sublevarlo. Como ya se ha dicho, en líneas generales estas prácticas son funcionales a esa producción tanto cultural como material, trasladando acríticamente significados e imposiciones atribuidas a cada sexo.

Estas limitaciones de nuestra profesión, compartida con otras también modernas, se hace más evidente cuando, como las/los autoras/os analizadas/os, nos deslizamos de una concepción del género como categoría analítica a una concepción del mismo como proceso activo que estructura múltiples aspectos de la vida social. Ninguna profesión es ingenua, entonces, al respecto. El trabajo social históricamente produce y reproduce prácticas de género y con género, no sólo respecto a aquellos con quien trabaja sino a la interna del propio colectivo profesional, donde aún pesa la invisibilidad de todo lo realizado, en general asociado a tareas de reproducción (Piriz, I994).

\section{La atribución de género. El ansia de clasificar}

En una línea cercana a Butler, Kessler y McKenna (I978) tratan de poner entre paréntesis las pretensiones ontológicas sobre género. Su objeto de estudio es el proceso de atribución de género, esa urgencia que rige la vida cotidiana por «clasificar» a las personas en uno u otro sexo y la inquietud ante lo ambiguo. Señalan, en la obra de referencia, que cada vez que la naturalización del género es problematizada, surgen nuevas formas que la revitalizan. Así, por ejemplo, la clasificación dicotómica entre hombres y mujeres según sus cuerpos sexuados ha sido a veces sustituida por un nuevo interés científico: la identidad de género, o sea, el sentido psicológico individual que se atribuye al ser hombre o mujer, como un fundamento más firme de una dicotomía fija.

Transexual es una categoría construida para poner de relieve la ambigüe- 
dad, para evitar los tipos de combinaciones (genitales masculinos e identidad de género femenina) que hacen que las personas se sientan incómodas porque violan reglas básicas sobre el género. Como actualmente se pueden cambiar los genitales, la identidad de género se ve ahora como un criterio menos flexible, marcando así el triunfo de los cirujanos sobre los psicoterapeutas en la urgencia por restaurar al género una realidad sin ambigüedad (Kessler y McKenna, I978: I 20).

En una postura también constructivista radical, Kessler y McKenna (I978) sostienen que el dimorfismo sexual no está dado por la naturaleza, sino que es impuesto a la naturaleza por quien lo percibe.

El género es una construcción social, un mundo de dos sexos es el resultado de los métodos compartidos socialmente y dados por hecho que las personas de un grupo social emplean para construir un mundo (Kessler y McKenna, I978: $\mathrm{x})$.

La concepción de géneros lleva a la percepción de dos sexos forzando a colocar todos los fenómenos percibidos dentro de las categorías dicotómicas postuladas. Anomalías y ambigüedades se ocultan, permitiendo que el fenómeno percibido se conforme y confirme la validez de la expectativa clasificatoria binaria.

Estos procesos que las autoras analizan en la vida cotidiana también modelan las percepciones socialmente legitimadas de los científicos, cuyos trabajos se toman como prueba incontrovertible de la validez de categorías y conceptos.

Los científicos construyen el dimorfismo allí donde hay continuidad. Hormonas, comportamientos, características físicas, procesos de desarrollo, cromosomas y cualidades psicológicas se han hecho encajar dentro de categorías dicotómicas. El conocimiento científico no informa la respuesta a la pregunta: ¿qué hace que una persona sea un hombre o una mujer? Más bien justifica (y parece que da bases a) la convicción ya existente de que una persona es o un hombre o una mujer y que no hay problemas para diferenciar entre ambos. Las diferencias biológicas, psicológicas y sociales no conducen a que veamos dos sexos. El hecho de que veamos dos sexos conduce al «descubrimiento» de diferencias biológicas, psicológicas y sociales (Kessler y McKenna, I978: I63).

De acuerdo con el supuesto ilustrado de que la biología proporciona el fundamento de lo masculino y lo femenino, es natural creer que la genitalidad brinda el criterio definitivo para la atribución de género. Pero las autoras indican que en la vida cotidiana, la atribución casi siempre se hace sin que haya ninguna confirmación sobre los genitales. A la que hace el médico a la hora 
del parto, sigue la asignación de sexo al nacimiento, pero en las interacciones sociales, la categorización del otro como hombre o mujer rara vez se basa en esa inspección directa. El énfasis en la identidad de género para continuar con tal clasificación dicotómica disloca la base de tal clasificación de la biología a los saberes «psi».

Cabe resaltar que, en líneas generales, el trabajo social realiza asignaciones dicotómicas de género, ya no sólo por esa actitud natural hacia el mismo sino también a partir de otras prácticas: encuentros visuales, análisis del contenido del habla y de la narrativa del self de los/las usuarios/as de los servicios, etcétera. Pero lo hace especialmente a partir de la constatación de los caracteres sexuales secundarios y terciarios de usarios/as, a las que estas autoras denominan «genitales culturales», lo que genera el reforzamiento de la actitud natural hacia el género.

Cuando los caracteres primarios y secundarios pueden ser modificados, la medicina solicita a los saberes $"$ psi» ${ }^{3}$ nueva bases para la reproducción dicotomizada del género: la identidad, como problemática entra en escena. Las profesiones asistenciales, y el trabajo social obviamente, juegan un papel secundario a la hora de definir categorías y criterios clasificatorios, pero aunque subordinado, es un papel fundamental a la hora de la circulación de sentidos sexuados.

\section{A modo de cierre}

Las propuestas teóricas sobre género que hemos analizado nos dicen que el género como explicación universal plantea graves problemas a los beneficios potenciales del género como concepto. Si las profesiones asistenciales van a confundir teóricamente el género con aquella actitud natural en lugar de ser confundidas por el género, sería útil que enriquecieran sus marcos analíticos, aprovechando distinciones cruciales como corporización sexuada o incardinamiento, sexualidad, identidad sexual, relaciones de trabajo y/o sociales marcadas por el género, simbolismos de género, experiencia situada (Beauvoir), órdenes e inventarios de género (Connell, 1987) en lugar de subsumir todo ello en un solo término: género.

Deberían tales profesiones - y no sólo ellas, como hemos visto en el caso

3. Para una comprensión de los saberes «psi» y sus funciones sociales, véase Donzelot (I986). 
de la medicina y los saberes «psi»— resistir firmemente la tendencia a tratar el género como explicación universal, a construirlo como una fuerza causal en terrenos tan dispares como la psiquis, el self, las relaciones sociales, en otras palabras, el cuerpo, el alma y la psiquis (Lamas, I994).

Las pretensiones universales sobre la invariabilidad del género o el papel desmesurado dado a la cultura en su producción, es decir, todo aquello que tiende a privatizarlo y no a verlo como agencia situada, significan la persistencia de la actitud natural, un fracaso sostenido que hay que superar.

Para ello no debemos justificar la ausencia de audacia teórica y práctica por la composición genérica - feminización - de los colectivos profesionales a los que hacemos referencia. Sería, como señala Grassi (2005), justificar la primera por tal condición. Esto constituiría no sólo un juicio injusto y sexista, sino teóricamente insostenible. Hablaría de una tendencia hacia la privatización de los dilemas y ausencias teórico-prácticas colectivas.

\section{Referencias}

Barros Cassal, Luan y Ana Lúcia Abrahão da Silva (20I0). Atravessamentos de Gênero e Produção de Subjetividade no Trabalho de Gestão de Nível Médio em Saúde. Revista Psicologia e Saúde, Vol. 2, Nro. I, Programa de Mestrado em Psicologia, UCDB, Campo Grande, MS, pp. I 8-23

Bordo, Susan (1993). Unbearable Weight. Feminism, Werstern Culture and the Body. Berkeley y Los Ángeles: University of California Press.

Butler, Judith (1990). Gender Trouble: Feminism and the Subversion of Identitiy. Nueva York y Londres: Routledge.

Connell, Robert W. (1987). Gender and Power. Stanford, California: Stanford University Press.

Chodonow, Nancy (1978). The Reproduction of Mothering. Berkeley: University of California Press.

Donzelot, Jaques (1986). A polícia das famílias. Río de Janeiro: Graal.

Femenías, María Luisa (2000). Sobre sujeto y género. Lecturas feministas desde Beauvoir a Butler. Buenos Aires: Catálogos.

Garfinkel, Harold (1967). Studies in Ethnomethodology. Englewood Cliffs, NJ: Prentice Hall.

Grassi, Estela (2005). «Prólogo». En La profesión de trabajo social, ¿̇cosa de mujeres? Estudios sobre el campo profesional desde la perspectiva de los 
trabajadores sociales. Genolet, Alicia y otros. UNER, Espacio Editorial. Buenos Aires, pp. 9-I4.

GiUliani, Delia (2006). "La práctica profesional del Trabajo Social y la inherente condición de lo femenino». UBA FCS. Disponible en <http://www. catedras.fsoc.uba.ar/ferraros/BD/DG\% 2opptsycdlf\% 20.pdf>.

Hawkesworth, Mary (г999). "Confundir el género». Debate Feminista, ıo (20).

Kessler, Suzanne y Wendy McKenna (1978). Gender: An Ethonomethodological Approach. Nueva York: Wiley.

KIng, Deborah (1988). «Multiple Jeopardy, Multiple Consciousness: The Context of a Black Feminist Ideology». Signs, I 4 (I): 42-72.

Lamas, Marta. (1994). «Cuerpo: diferencia social y género». Debate Feminista, 5 (Io): 3-3I.

PIriz, Clara (1994). «Profesión Clase B de ciudadanas de segunda». En Varios autores, Demandas y oportunidades para el trabajo social (pp. 2I3-237). Montevideo: Eppal.

Rebollo, M. de los Ángeles, Eva González y M. del Rosario García (s/f). «Identidades profesionales en educación desde una perspectiva de género». Disponible en <http://www.revistafuentes.es/gestor/apartados_revista/pdf/ numeros_anteriores/nnwfjcdd.pdf $>$.

SARTre, Jean Paul (2004). «Cuestiones de método». Apéndice. En Crítica de la razón dialéctica. Vol. I (pp. I 5-I 55 ). Buenos Aires: Losada.

Spelman, Elizabeth (1988). Inessential Woman. Boston: Beacon.

Staton, Donna (1980). «Lenguage and Revolution: The Franco-American Disconnection». En H. Eisten y A. Jardin (eds.), The Future Difference. Boston: G.K. Hall.

\section{Sobre la autora}

Mónica de Martino es académica de la Universidad de la República del Uruguay. Trabajadora Social, doctora en Ciencias Sociales por la Universidad Estadual de Campinas, Brasil. Su correo electrónico es < monica.demartino@ cienciassociales.edu.uy>. 